\title{
The Impact of Antidumping Policy of US Biodiesel Import on Indonesia and Argentina Economy - A General Equilibrium Analysis
}

\author{
Salman Samir and Samsubar Saleh
}

\begin{abstract}
World markets tend to be more free and open. This phenomenon is unavoidable because every country wants a world market that is open to its export products. Every obstacle, both tariff and non-tariff, is attempted to be reduced or eliminated through bilateral, regional and multilateral agreements. While countries expect an increasingly free world market, the United States (US) withdraws and becomes protectionist. In April 2018, the US officially decided on the implementation of antidumping import tariffs for Argentine and In donesian biodiesel products. The US raised antidumping import tariffs, after accusing the two countries of dumping practices. The tariff policy for imports of antidumping us seeks to protect domestic industries. This study aims to analyze the impact of US antidumping import tariffs on the economies of Indonesia and Argentina. This study uses the Computable General Equilibrium (CGE) model to observe the impact of rising US antidumping import tariffs on the economies of Indonesia and Argentina and other regions. The results of this study found that US antidumping import tariff policies have a negative impact on the economy of Indonesia and Argentina. In addition, the policy also has a negative impact on countries in the Asia-Pacific region and the EU. This shows that the implementation of US antidumping import tariffs for Indonesian and Argentinian biodiesel products has a global impact.
\end{abstract}

Index Terms-International economy, Antidumping, Computable General Equilibrium

\section{INTRODUCTION}

The protectionist phenomenon of developed countries has become a concern in recent years [1]. One of the most popular forms of protectionis $\mathrm{m}$ and import protection policy is the antidumping policy. An antidumping policy becomes a country strategy for securing domestic industries [2]. [3] stated that the antidumping policy was basically justified as a form of protection against exporters who were dumping. He said since the mid-1970s, antidumping emerged as a form of "new protectionism". The antidumping is an instrument that can be used by member countries of World Trade Organization (WTO) to secure its domestic industry from the consequences of unnatural selling price in the form of loss to the domestic industry producing similar goods. In this regard, the WTO permits members whose industries suffer losses as a result of the unusual sale price, to carry out antidumping actions, in the form of imposition of antidumping duties other than normal import duty [4]. This

Manuscript received September 8, 2018; revised November 12, 2018.

Salman Samir is with Magister Program in Economics, Universitas Gadjah Mada, Indonesia (e-mail: salmansamirmsc@gmail.com).

Samsubar Saleh is with Faculty of Economics and Business, Universitas Gadjah Mada, Indonesia phenomenon is no longer confined to a few developed countries; Some developing and developing countries have started using antidumping policies aggressively [5]. According to [6] there seems to be a genuine concern for maintaining the competitive environment in the domestic economy, as a retaliatory tool against other countries, providing protection to the domestic industries, political factors, etc. In this regard, antidumping policy becomes a necessity for a country in facing global competition.

While countries expect an increasingly free world trade, the United States (US) withdraws and becomes protectionist [7]. Proven in 2015 and 2016, the US changed antidumping and award (antisubsidized) provisions. Amendments to the anti-dumping and benefits provisions are the Selective Renewal of Trade Act (TPE) in June 2015 and the Trade and Trade Facilitation Act (TFTE) in February 2016. This amendment is based on proposals from the US domestic industry. The TPE amendment makes it easy for the US domestic industry. The US industry can claim losses from imports by banning the US government from declaring the domestic industry to be unprofitable for imports, simply because the industry has benefited in recent years. In addition, TFTE amend ments provide for the US govern ment in implementing dumping decisions for exporters [8].

Based on World Trade Organization (WTO) data (see figure 1), the number of antidumping cases in the US from 2014 to 2017 rose in significance. The amount of antidumping cases in 2014 is 4 cases, 7 cases in 2015, 14 cases in 2016 and 34 cases in 2017.

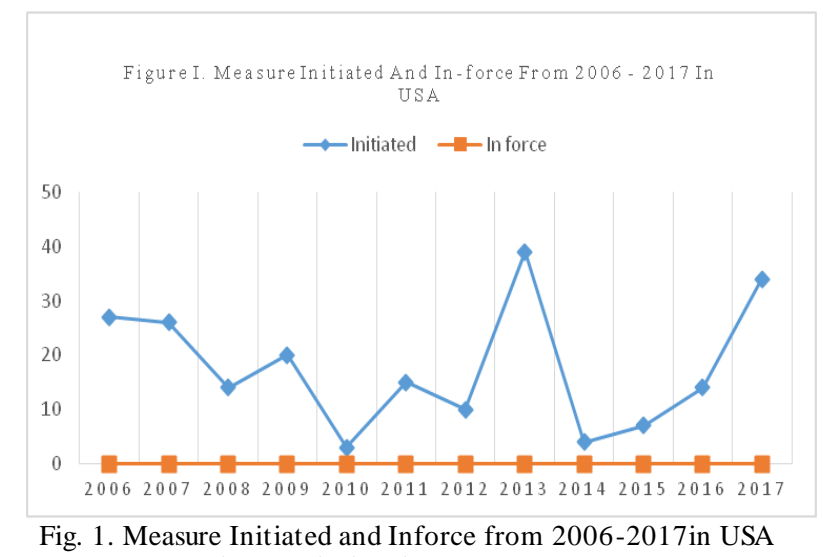

Source: Authors' calculated results from WTO dataset.

In April 2018, the US has announced the imposition of antidumping duties of 126.97 percent to 341.38 percent for biodiesel originating from Argentina and Indonesia. The US raised antidumping duties of biodiesel products from Indonesia and Argentina, after accusing the two countries of dumping practices (setting export prices below domestic 
market prices). The imposition of antidu mping duties earlier in 2017 of about 50 to 70 percent is imposed before US finds evidence of biodiesel sales below the prevailing market price in the US. In detail, Argentine-based biodiesel made from soybean oil is exposed to antidumping duties 54.36 to 70.05 percent. The antidumping duties for Indonesia's biodiesel, based on oil palm reached 50.71 percent [9].

This study aims to analyze the impact of antidu mping us import tariffs on the economies of Indonesia and Argentina. This study uses a counterfactual approach in assessing the impact of antidumping policies not only on the three countries involved but also for other biodiesel production countries that collaborate with the US such on others country. The remainder of the paper is divided into the following sections. Section II discusses the previous research. Section III discusses the research methodology. Section IV is followed by an results and analysis. Finally, in section $\mathrm{V}$, we conclude.

\section{PREVIOUS RESEARCH}

The study of antidumping policies has been carried out in both developed and developing countries. The focus of the study on antidumping policies depends on the problems faced by each country. Differences in analysis units, periods, analytical tools, and the use of instruments in various studies can produce different conclusions and policy recommendations. For example, Webb's 1992 study which found that the imposition of antidumping import tariffs would reduce the volume of domestic imports and increase the profits of domestic producers [10]. The study [11] analyzed the impact of antidumping import tariff policies on the welfare and industrial output of exporters. The results of the study found that in the short term, an increase in the import tariff for antidumping would significantly reduce the output of exporters. In the long run, the decline in industrial output causes prosperity.

The study [12] revealed the negative impact of the antidumping policy on the exporter industry. It is different from [13] which observes changes in exports in response to the possibility of imposing antidumping import tariffs. According to him, there is a quick response by exporters to the possibility of applying antidumping import tariffs by importers. This response is shown by the shift of export of goods and services to other importers (trade depletion). Several studies have used the CGE models for analysis impact trade policy. The for example, CGE models are used by [14] to assessing the trade impacts of these Non-Tarif Measures (NTMs) under the ASEAN Free Trade Agreement (AFTA).

\section{RESEARCH METHODOLOGY}

This study uses the CGE model assuming a perfectly competitive market and constant returns to scale. This study uses Global Trade Analysis Projects (GTAP). GTAP with the latest database of 140 regions, 57 industries and five production factors for each region allows analyzing the impact of US antidumping import tariff policies on industrial output, export volume, Gross Domestic Product (GDP) and Indonesian and Argentinian welfare. The method of the disaggregation is detailed in Table 1 and Table 2.

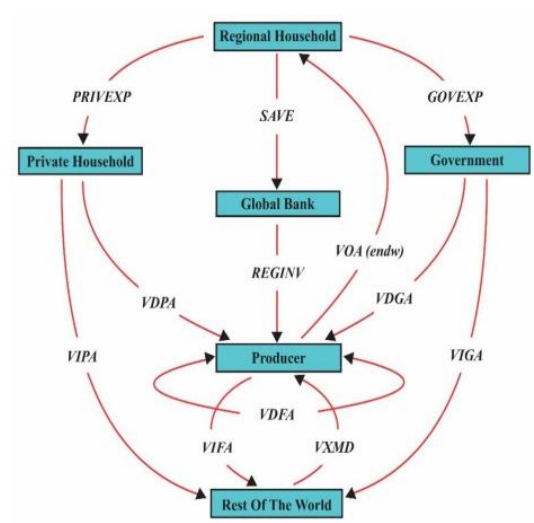

Fig. 2. Graphical representation of a GT AP model. Source: [15].

T ABLE I: THE AgGREgation OF THE TwENTY Two REgIONS

\begin{tabular}{cccc}
\hline \hline No & $\begin{array}{c}\text { Region } \\
\text { code }\end{array}$ & $\begin{array}{c}\text { Comprising } \\
\text { economies }\end{array}$ & Description \\
\hline 1 & ARG & Argentina & Argentina \\
2 & CAN & Canada & Canada \\
3 & US & United States & United States \\
4 & PAN & Panama & Panama \\
5 & NOR & Norway & Norway \\
6 & NLD & Netherlands & Netherlands \\
7 & DEU & Germany & Germany \\
8 & BEL & Belgium & Belgium \\
9 & FIN & Finlandia & Finlandia \\
10 & PRT & Portugal & Portugal \\
11 & ESP & Spain & Spain \\
12 & IDN & Indonesia & Indonesia \\
13 & AUS & Australia & Australia \\
14 & SGP & Singapore & Singapore \\
15 & KOR & Korea & Korea \\
16 & TWN & Taiwan & Taiwan \\
17 & CHN & China & China \\
18 & NA & North America & North America \\
19 & LA & Latin America & Latin America \\
20 & AP & Asia Pacific & Asia Pacific \\
21 & UE & European Union & The 21 EU countries in \\
22 & RAW & Rest of the World & All other countries that \\
& & & are not included in the 5 \\
& & & groups \\
\hline \hline
\end{tabular}

T ABLE II: THE AGGREGATION OF THE TWELVE INDUSTRY

\begin{tabular}{|c|c|c|}
\hline No & Industry code & Comprising economies \\
\hline 1 & $\begin{array}{l}\text { Vegetable } \\
\text { Oils }\end{array}$ & Vegetable Oils \\
\hline 2 & Oil Seeds & $\begin{array}{l}\text { Oil seeds and oleaginous fruit; soy beans, } \\
\text { copra }\end{array}$ \\
\hline 3 & $\begin{array}{l}\text { Grain and } \\
\text { crops }\end{array}$ & $\begin{array}{l}\text { Paddy rice, wheat, cereal grain, vegetable, } \\
\text { fruits, nuts, beet, crops, plant-based fibbers, } \\
\text { wool, silk-worm cocoons, forestry, sugar cane, }\end{array}$ \\
\hline 4 & Meat & $\begin{array}{l}\text { Cattle, sheep, goat, horses, animal product, } \\
\text { fishing, meat, dairy product, }\end{array}$ \\
\hline 5 & Extraction & $\begin{array}{l}\text { Mining and Extraction Coal oil gas fish } \\
\text { minerals, }\end{array}$ \\
\hline 6 & $\begin{array}{l}\text { Processing } \\
\text { food }\end{array}$ & sugar, milk, beverage, and tobacco \\
\hline 7 & Textiles & Textiles and apparel \\
\hline 8 & $\begin{array}{l}\text { Light } \\
\text { manufacture }\end{array}$ & $\begin{array}{l}\text { Leather, wood products, paper, publishing } \\
\text { metal, motor vehicles transport equipment, } \\
\text { manufactures nec }\end{array}$ \\
\hline 9 & $\begin{array}{l}\text { Heavy } \\
\text { Manufacture }\end{array}$ & $\begin{array}{l}\text { Petroleum, coal, chemical rubber, plastic, } \\
\text { mineral product nec. ferrous metals, metals } \\
\text { electronic equipment }\end{array}$ \\
\hline 10 & $\begin{array}{l}\text { Constructions } \\
\text { and utilities }\end{array}$ & $\begin{array}{l}\text { Electricity, gas distribution, water, } \\
\text { construction }\end{array}$ \\
\hline 11 & $\begin{array}{l}\text { Transport and } \\
\text { communicatio } \\
\mathrm{n}\end{array}$ & metals, metals electronic equipment \\
\hline 12 & Other Service & $\begin{array}{l}\text { Financial, insurance, business service, } \\
\text { recreational, public service }\end{array}$ \\
\hline
\end{tabular}

Fig. 2 displays its most fundamental structure for a 
representative region or country. The regional household is linked with the producer or firm through private household expenditure (PRIVEXP) and government expenditure (GOVEXP). The producers receive payments for selling consumption goods to the private households (VDPA) and the government (VDGA), intermediate inputs to other producers (VDFA) and investment goods to the savings sector (NETINV). Under the zero profit assumption employed in GTAP, these revenues must be precisely exhausted on expenditures for intermed iate inputs (VDFA) and primary factors of production (VOA). While savings are completely exhausted on investment (NETINV) through a global bank, the producer is linked with the rest of the world through exports and import of both the intermediate inputs (VXMD and VIFA) and the final product (VIPA and VIGA). The GTAP model employs the so-called Armington assumption in the trading sector which provides the possibility to distinguish imports by their origin and explains the intra-industry trade of similar product [15].

In this paper, 3 scenarios are prepared to look at the economic changes in each country:

1.Baseline: When both countries are subject to a normal import tariff for biodiesel products of 6.5 percent (source: United States International Trade Commission (USITC)). 2.The scenario of tariffs of 127 percent (minimum tariffs) for biodiesel products, imposed after the US announced the imposition of antidumping duties for both countries.

3. Scenario tariffs 341 percent (maximum tariffs).

4. In detail, Argentine-based biodiesel made from soybean oil is exposed to antidumping duties. The antidumping duties for Indonesia's biodiesel, which is based on oil palm.

T ABLE III: CHANGES IN INDUSTRY OUTPUT(\%)

\begin{tabular}{|c|c|c|c|c|c|c|c|c|c|c|c|c|c|c|c|c|c|c|c|c|c|c|}
\hline & & & & & & & & & ariffs 12 & scenar & for case & donesia & & & & & & & & & & \\
\hline Region & ARG & CAN & US & PAN & NOR & $\mathrm{NLD}$ & $\mathrm{DEU}$ & BEL & FN & PRT & ESP & $\mathbb{D N N}$ & AUS & SGP & KOR & TWN & CHN & $\mathrm{NA}$ & LA & AP & UE & RAW \\
\hline vegetable_oi & 6.33 & 1.87 & 2.63 & 2.17 & 0.86 & 1.50 & 2.18 & 2.21 & 1.78 & 2.39 & 2.01 & -35.78 & 4.83 & 12.17 & 1.76 & 1.09 & 0.91 & 0.10 & 2.22 & 12.99 & 1.32 & 4.66 \\
\hline GrainsCrops & 0.51 & 0.24 & 0.13 & 0.08 & 0.05 & 0.20 & 0.13 & 0.17 & 0.05 & 0.15 & 0.15 & -0.91 & -0.03 & -0.04 & 0.03 & 0.06 & 0.08 & 0.06 & 0.14 & 0.33 & 0.10 & 0.08 \\
\hline Meat & -0.49 & 0.02 & 0.00 & 0.01 & 0.03 & 0.03 & 0.03 & 0.03 & 0.04 & 0.01 & -0.01 & 0.67 & 0.06 & 0.04 & 0.01 & 0.01 & -0.01 & 0.01 & -0.01 & -0.11 & 0.02 & 0.01 \\
\hline Exraction & -0.35 & -0.01 & -0.01 & -0.02 & 0.00 & -0.01 & 0.00 & -0.03 & -0.01 & -0.02 & -0.03 & 0.82 & -0.01 & 0.03 & 0.01 & 0.00 & 0.00 & -0.01 & -0.05 & -0.02 & -0.01 & -0.01 \\
\hline ProcFood & -0.29 & 0.01 & 0.00 & 0.03 & 0.04 & 0.02 & 0.00 & 0.01 & -0.01 & 0.01 & -0.01 & 0.91 & 0.01 & 0.10 & -0.01 & 0.01 & -0.01 & 0.01 & -0.02 & -0.14 & 0.01 & 0.01 \\
\hline TextWapp & -0.44 & -0.04 & -0.04 & 0.01 & 0.01 & -0.02 & 0.00 & -0.01 & 0.01 & -0.02 & -0.03 & 3.69 & -0.01 & 0.19 & 0.04 & 0.03 & -0.03 & -0.05 & -0.10 & -0.13 & -0.01 & -0.05 \\
\hline LightMnfe & -0.68 & -0.04 & -0.02 & -0.04 & 0.00 & -0.03 & -0.01 & -0.02 & -0.01 & -0.02 & -0.02 & 2.02 & -0.03 & -0.10 & 0.01 & -0.01 & -0.02 & 0.01 & -0.02 & -0.10 & -0.02 & -0.04 \\
\hline HeavyMnfc & -0.62 & -0.02 & -0.02 & -0.03 & 0.01 & -0.01 & -0.01 & -0.02 & 0.00 & -0.01 & -0.02 & 3.12 & -0.02 & -0.03 & -0.01 & -0.01 & -0.01 & -0.01 & -0.07 & -0.10 & -0.01 & -0.03 \\
\hline Util_Cons & 0.58 & 0.00 & 0.00 & 0.00 & -0.01 & -0.01 & -0.01 & -0.01 & -0.01 & -0.01 & -0.01 & -0.12 & 0.00 & 0.01 & -0.01 & -0.01 & -0.02 & -0.01 & 0.01 & -0.02 & -0.01 & -0.01 \\
\hline TransComm & 0.06 & 0.00 & 0.00 & -0.01 & -0.01 & -0.01 & 0.00 & -0.01 & 0.00 & -0.01 & 0.00 & 0.28 & 0.00 & 0.00 & 0.00 & 0.00 & -0.01 & 0.00 & 0.00 & -0.01 & -0.01 & 0.00 \\
\hline OthServices & 0.03 & 0.00 & 0.00 & 0.00 & 0.00 & 0.00 & 0.00 & 0.00 & 0.00 & 0.00 & 0.00 & 0.06 & 0.00 & 0.01 & 0.00 & 0.00 & 0.00 & 0.00 & 0.00 & -0.03 & 0.00 & -0.01 \\
\hline
\end{tabular}

\begin{tabular}{|c|c|c|c|c|c|c|c|c|c|c|c|c|c|c|c|c|c|c|c|c|c|c|}
\hline \multicolumn{23}{|c|}{ tariffs $341 \%$ scenario for case Indonesia } \\
\hline Region & ARG & CAN & US & PAN & NOR & $\mathrm{NLD}$ & DEU & BEL & FN & PRT & ESP & IDN & AUS & SGP & KOR & TWN & CHN & $\mathrm{NA}$ & LA & AP & UE & RAW \\
\hline vegetable_oi & 8.34 & 3.24 & 3.65 & 3.18 & 1.73 & 3.26 & 4.24 & 4.41 & 3.76 & 4.97 & 3.74 & -38.82 & 5.86 & 15.60 & 2.15 & 1.18 & 1.83 & 0.18 & 3.07 & 19.48 & 2.39 & 7.80 \\
\hline Grains Crops & 0.72 & 0.45 & 0.25 & 0.14 & 0.10 & 0.42 & 0.29 & 0.35 & 0.10 & 0.29 & 0.31 & -0.29 & 0.19 & 0.24 & 0.06 & 0.11 & 0.17 & 0.15 & 0.25 & 0.47 & 0.21 & 0.15 \\
\hline Meat & -0.67 & 0.03 & -0.01 & 0.02 & 0.05 & 0.03 & 0.04 & 0.05 & 0.05 & 0.01 & -0.03 & 0.38 & 0.05 & 0.07 & 0.01 & 0.01 & -0.01 & 0.01 & -0.01 & -0.15 & 02 & 0.01 \\
\hline Exraction & -0.50 & -0.03 & -0.02 & -0.05 & -0.01 & -0.02 & -0.02 & -0.07 & -0.03 & -0.07 & -0.08 & 1.04 & -0.03 & 0.01 & -0.03 & -0.02 & -0.02 & -0.03 & -0.08 & 0.02 & -0.02 & -0.02 \\
\hline ProcFood & -0.39 & 0.02 & 0.00 & 0.04 & 0.07 & 0.04 & -0.01 & 0.01 & -0.03 & 0.02 & -0.03 & 1.59 & 0.01 & -0.08 & -0.03 & 0.00 & -0.02 & 0.01 & -0.03 & -0.19 & 0.01 & 0.02 \\
\hline TextWapp & -0.60 & -0.06 & -0.07 & 0.04 & 0.01 & -0.04 & -0.01 & -0.04 & 0.01 & -0.05 & -0.06 & 2.68 & -0.01 & 0.33 & 0.02 & 0.02 & -0.06 & -0.05 & -0.13 & -0.23 & -0.02 & -0.07 \\
\hline LightMnfc & -0.93 & -0.07 & -0.03 & -0.06 & 0.00 & -0.05 & -0.01 & -0.03 & -0.01 & -0.05 & -0.04 & 1.36 & -0.04 & -0.12 & 0.02 & 0.00 & -0.03 & 0.01 & -0.03 & -0.18 & -0.03 & -0.05 \\
\hline HeavyMnfc & -0.84 & -0.04 & -0.03 & -0.07 & 0.02 & -0.03 & -0.02 & -0.04 & 0.00 & -0.02 & -0.04 & 2.45 & -0.03 & -0.05 & -0.02 & -0.0 & -0.02 & -0.01 & -0.11 & -0.17 & -0.02 & -0.05 \\
\hline Util_Cons & 0.80 & 0.00 & 0.01 & 0.00 & -0.01 & -0.01 & -0.01 & -0.01 & -0.02 & 0.00 & 0.00 & -0.30 & 0.00 & 0.00 & -0.01 & 0.00 & -0.02 & -0.01 & 0.02 & -0.05 & -0.01 & -0.01 \\
\hline TransConm & 0.07 & 0.00 & 0.00 & -0.03 & 0.00 & -0.01 & 0.00 & -0.01 & -0.01 & -0.01 & -0.01 & 0.12 & 0.00 & 0.02 & 0.00 & 0.00 & -0.01 & 0.00 & 0.00 & -0.03 & -0.01 & -0.01 \\
\hline OthServices & 0.04 & 0.00 & 0.00 & 0.00 & 0.00 & -0.01 & 0.00 & -0.01 & 0.00 & -0.01 & -0.01 & -0.03 & 0.00 & 0.02 & 0.00 & 0.00 & -0.01 & 0.00 & -0.01 & -0.07 & 0.00 & -0.01 \\
\hline
\end{tabular}

Source: Authors' results from GTAP simulation

\begin{tabular}{|c|c|c|c|c|c|c|c|c|c|c|c|c|c|c|c|c|c|c|c|c|c|c|}
\hline \multicolumn{23}{|c|}{ tariiffs $127 \%$ scenario for case Argentina } \\
\hline oilseeds & -2.54 & 0.47 & 0.50 & 0.26 & 0.53 & -0.01 & 0.05 & 0.06 & 0.01 & 0.07 & 0.06 & 0.04 & 0.19 & 0.13 & 0.07 & 0.33 & 0.24 & 0.19 & 0.54 & 0.12 & 0.07 & 0.17 \\
\hline GrainsCrops & 1.08 & -0.04 & -0.04 & -0.02 & -0.02 & -0.02 & -0.01 & -0.02 & 0.00 & -0.02 & -0.02 & -0.01 & -0.01 & -0.01 & 0.00 & 0.00 & -0.01 & 0.00 & -0.04 & -0.01 & -0.01 & -0.01 \\
\hline Meat & 0.25 & -0.01 & -0.01 & 0.00 & 0.00 & 0.00 & 0.00 & -0.01 & 0.00 & 0.00 & 0.00 & 0.00 & 0.00 & 0.00 & 0.00 & 0.00 & 0.00 & 0.00 & -0.01 & 0.00 & 0.00 & 0.00 \\
\hline Extraction & 0.09 & 0.00 & 0.00 & 0.00 & 0.00 & 0.00 & 0.00 & 0.00 & 0.00 & 0.00 & 0.00 & 0.00 & 0.00 & 0.00 & 0.00 & 0.00 & 0.00 & 0.00 & 0.00 & 0.00 & 0.00 & 0.00 \\
\hline ProcFood & 0.11 & 0.00 & 0.00 & 0.01 & 0.00 & 0.00 & 0.00 & 0.00 & 0.00 & 0.00 & 0.00 & 0.00 & 0.00 & 0.00 & 0.00 & 0.00 & -0.01 & 0.00 & -0.01 & 0.00 & 0.00 & 0.00 \\
\hline TextWapp & 0.14 & 0.00 & 0.00 & 0.01 & 0.00 & 0.01 & 0.01 & 0.01 & 0.00 & 0.01 & 0.01 & 0.01 & 0.00 & 0.01 & 0.01 & 0.01 & -0.01 & 0.00 & -0.01 & 0.00 & 0.00 & 0.00 \\
\hline LightMnfc & 0.20 & -0.01 & 0.00 & 0.00 & 0.00 & 0.00 & 0.00 & 0.00 & 0.00 & 0.00 & 0.00 & 0.00 & 0.00 & 0.00 & 0.00 & 0.00 & 0.00 & 0.00 & -0.02 & 0.00 & 0.00 & 0.00 \\
\hline HeavyMnfc & 0.15 & 0.00 & 0.00 & 0.01 & 0.00 & 0.00 & 0.00 & 0.00 & 0.00 & 0.00 & 0.00 & 0.00 & 0.00 & 0.00 & 0.00 & 0.00 & 0.00 & 0.00 & -0.01 & 0.00 & 0.00 & 0.00 \\
\hline Util_Cons & -0.12 & 0.00 & 0.00 & 0.00 & 0.00 & 0.00 & 0.00 & 0.00 & 0.00 & 0.00 & 0.00 & 0.00 & 0.00 & 0.00 & 0.00 & 0.00 & 0.00 & 0.00 & 0.01 & 0.00 & 0.00 & 0.00 \\
\hline TransComm & 0.03 & 0.00 & 0.00 & 0.00 & 0.00 & 0.00 & 0.00 & 0.00 & 0.00 & 0.00 & 0.00 & 0.00 & 0.00 & 0.00 & 0.00 & 0.00 & 0.00 & 0.00 & 0.00 & 0.00 & 0.00 & 0.00 \\
\hline OthServices & -0.02 & 0.00 & 0.00 & 0.00 & 0.00 & 0.00 & 0.00 & 0.00 & 0.00 & 0.00 & 0.00 & 0.00 & 0.00 & 0.00 & 0.00 & 0.00 & 0.00 & 0.00 & 0.00 & 0.00 & 0.00 & 0.00 \\
\hline
\end{tabular}

\begin{tabular}{|c|c|c|c|c|c|c|c|c|c|c|c|c|c|c|c|c|c|c|c|c|c|c|}
\hline \multicolumn{23}{|c|}{ tariffs $341 \%$ scenario for case Argentina } \\
\hline Region & ARG & CAN & US & PAN & NOR & NLD & DEU & BEL & FIN & PRT & ESP & IDN & AUS & SGP & KOR & TWN & $\mathrm{CHN}$ & $\mathrm{NA}$ & LA & $\mathrm{AP}$ & $\mathrm{UE}$ & RAW \\
\hline oilseeds & -6.07 & 1.07 & 1.24 & 0.69 & 0.93 & -0.01 & 0.12 & 0.15 & 0.02 & 0.17 & 0.14 & 0.09 & 0.45 & 0.28 & 0.17 & 0.61 & 0.60 & 0.46 & 1.34 & 0.23 & 0.16 & 0.43 \\
\hline GrainsCrops & 2.56 & -0.08 & -0.10 & -0.04 & -0.04 & -0.04 & -0.03 & -0.06 & -0.01 & -0.05 & -0.05 & -0.02 & -0.03 & -0.03 & 0.00 & 0.00 & -0.02 & 0.01 & -0.10 & -0.02 & -0.03 & -0.03 \\
\hline Meat & 0.59 & -0.02 & -0.02 & 0.01 & 0.00 & -0.01 & -0.01 & -0.01 & 0.00 & 0.00 & 0.00 & 0.00 & 0.00 & 0.01 & 0.00 & 0.00 & -0.01 & 0.00 & -0.03 & 0.00 & 0.00 & -0.01 \\
\hline Extraction & 0.20 & 0.00 & 0.00 & 0.00 & 0.00 & 0.00 & 0.00 & 0.00 & 0.00 & 0.00 & 0.00 & 0.00 & 0.00 & 0.00 & 0.00 & 0.00 & 0.00 & 0.00 & -0.01 & 0.00 & 0.00 & 0.00 \\
\hline ProcFood & 0.26 & -0.01 & -0.01 & 0.02 & 0.00 & 0.00 & 0.00 & 0.00 & 0.00 & 0.00 & 0.00 & 0.01 & 0.00 & 0.01 & -0.01 & -0.01 & -0.01 & 0.00 & -0.01 & 0.00 & 0.00 & 0.00 \\
\hline TextWapp & 0.33 & 0.00 & 0.00 & 0.03 & 0.01 & 0.01 & 0.01 & 0.01 & 0.01 & 0.01 & 0.01 & 0.03 & 0.01 & 0.02 & 0.01 & 0.02 & -0.02 & 0.01 & -0.01 & 0.01 & 0.01 & 0.01 \\
\hline LightMnfc & 0.48 & -0.01 & -0.01 & -0.01 & 0.00 & 0.00 & 0.00 & 0.00 & 0.00 & 0.00 & 0.00 & 0.00 & 0.00 & 0.00 & 0.00 & 0.00 & 0.00 & 0.00 & -0.04 & 0.00 & 0.00 & 0.00 \\
\hline HeavyMnfc & 0.34 & -0.01 & -0.01 & 0.01 & 0.00 & 0.00 & 0.00 & 0.00 & 0.00 & 0.00 & 0.00 & 0.00 & 0.00 & 0.00 & 0.00 & 0.00 & 0.00 & 0.00 & -0.02 & 0.00 & 0.00 & 0.00 \\
\hline Util_Cons & -0.28 & 0.00 & 0.01 & 0.00 & 0.00 & 0.00 & 0.00 & 0.00 & 0.00 & 0.00 & 0.00 & 0.00 & 0.00 & 0.00 & 0.00 & 0.00 & -0.01 & 0.00 & 0.01 & 0.00 & 0.00 & 0.00 \\
\hline TransConm & 0.07 & 0.00 & 0.00 & 0.00 & 0.00 & 0.00 & 0.00 & 0.00 & 0.00 & 0.00 & 0.00 & 0.00 & 0.00 & 0.00 & 0.00 & 0.00 & 0.00 & 0.00 & 0.00 & 0.00 & 0.00 & 0.00 \\
\hline OthServices & -0.04 & 0.00 & 0.00 & 0.00 & 0.00 & 0.00 & 0.00 & 0.00 & 0.00 & 0.00 & 0.00 & 0.00 & 0.00 & 0.00 & 0.00 & 0.00 & 0.00 & 0.00 & 0.00 & 0.00 & 0.00 & 0.00 \\
\hline
\end{tabular}

Source: Authors' results from GT AP simulation 
TABLE IV: CHANGE IN REGIONAL GDP (\%)

\begin{tabular}{|c|c|c|c|c|c|c|}
\hline \multirow{2}{*}{ Region } & \multicolumn{3}{|c|}{ Tarif Import on Indonesia } & \multicolumn{3}{|c|}{ Tarif Import on Argentina } \\
\hline & Baseline & Tariffs $127 \%$ & Tariffs $341 \%$ & Baseline & Tariffs $127 \%$ & Tariffs $341 \%$ \\
\hline Argentina & 0,001 & 0,600 & 0,833 & $-0,010$ & $-0,170$ & $-0,398$ \\
\hline Canada & 0,004 & 0,014 & 0,028 & 0,000 & 0,004 & 0,008 \\
\hline US & 0,005 & 0,013 & 0,028 & 0,000 & 0,003 & 0,007 \\
\hline Panama & 0,045 & 0,020 & 0,038 & 0,000 & $-0,001$ & $-0,001$ \\
\hline Norway & 0,001 & $-0,002$ & $-0,002$ & 0,000 & 0,000 & 0,000 \\
\hline Netherlands & 0,001 & 0,005 & 0,018 & 0,000 & 0,000 & 0,001 \\
\hline Germany & 0,000 & 0,002 & 0,007 & 0,000 & 0,001 & 0,001 \\
\hline Belgium & $-0,130$ & 0,008 & 0,022 & 0,000 & 0,000 & 0,000 \\
\hline Finlandia & 0,000 & 0,000 & 0,004 & 0,000 & 0,000 & 0,001 \\
\hline Spain & 0,000 & 0,013 & 0,029 & 0,000 & 0,000 & 0,000 \\
\hline Indonesia & 0,001 & $-1,468$ & $-1,723$ & 0,000 & 0,001 & 0,002 \\
\hline Australia & 0,000 & $-0,001$ & 0,005 & 0,000 & 0,001 & 0,002 \\
\hline Singapore & 0,000 & $-0,007$ & $-0,017$ & 0,000 & 0,000 & 0,001 \\
\hline Korea & 0,001 & $-0,005$ & 0,002 & 0,000 & 0,000 & 0,000 \\
\hline Taiwan & 0,000 & $-0,003$ & 0,000 & 0,000 & 0,000 & 0,001 \\
\hline China & 0,001 & $-0,001$ & 0,004 & 0,000 & 0,000 & 0,000 \\
\hline North America & 0,000 & 0,007 & 0,015 & 0,000 & 0,000 & 0,001 \\
\hline Latin America & 0,001 & 0,061 & 0,094 & 0,000 & 0,006 & 0,015 \\
\hline Asia Pacific & $-0,001$ & 0,013 & $-0,109$ & 0,000 & 0,001 & 0,001 \\
\hline RAW & 0,001 & 0,017 & 0,028 & 0,000 & 0,001 & 0,001 \\
\hline
\end{tabular}

Source: Authors' results from GT AP simulations

TABEL V: REGIONAL WELFARE CHANGE (EQUIVALENT VARIATION US\$MILLION).

\begin{tabular}{|c|c|c|c|c|c|c|}
\hline \multirow{2}{*}{ Region } & \multicolumn{3}{|c|}{ Indonesia } & \multicolumn{3}{|c|}{ Argentina } \\
\hline & Baseline & tariffs $127 \%$ & tariffs $341 \%$ & Baseline & tariffs $127 \%$ & tariffs $341 \%$ \\
\hline Argentina & 41.423 & 549.904 & 761.295 & -11.876 & -210.434 & -488.972 \\
\hline Canada & 0.037 & 6.064 & 16.506 & 0.547 & 9.087 & 19.398 \\
\hline US & 20.444 & 287.97 & 539.034 & 3.460 & 65.108 & 163.371 \\
\hline Panama & 0.138 & 2.497 & 5.255 & -0.013 & -0.216 & -0.477 \\
\hline Norway & -1.45 & -15.099 & -33.681 & 0.000 & -0.604 & -2.298 \\
\hline Netherlands & -0.756 & -6.657 & 5.66 & 0.027 & 0.428 & 0.842 \\
\hline Germany & -4.394 & -58.879 & -100.638 & 0.297 & 5.103 & 11.289 \\
\hline Belgium & 0.225 & 6.349 & 16.529 & -0.044 & -0.812 & -2.029 \\
\hline Finlandia & -0.385 & -6.514 & -11.306 & 0.012 & 0.199 & 0.429 \\
\hline Portugal & -0.365 & -5.37 & -6.778 & 0.016 & 0.275 & 0.588 \\
\hline Spain & 0.813 & 10.49 & 20.31 & 0.151 & 2.601 & 5.806 \\
\hline Indonesia & -163.834 & -1813.24 & -2557.24 & 0.154 & 2.605 & 5.494 \\
\hline Australia & -1.377 & -2.787 & -8.196 & 0.008 & 0.08 & -0.046 \\
\hline Singapore & 1.581 & 24.359 & -0.811 & -0.059 & -1.03 & -2.38 \\
\hline Korea & -2.93 & -33.22 & -6.203 & -0.520 & -9.704 & -23.569 \\
\hline Taiwan & -1.044 & -9.656 & 3.359 & -0.086 & -1.603 & -4.005 \\
\hline China & -23.699 & -310.451 & -660.997 & -0.501 & -21.062 & -98.603 \\
\hline North America & -1.326 & -13.834 & -27.522 & -0.274 & -5.088 & -12.645 \\
\hline Latin America & 12.175 & 164.569 & 261.869 & 3.968 & 73.405 & 180.471 \\
\hline
\end{tabular}




\begin{tabular}{lrrrrrr} 
Asia Pacific & -250.977 & -4332.53 & -12355.6 & -0.243 & -11.2 & -38.457 \\
UE & -6.666 & -77.435 & -161.087 & 0.794 & 13.951 & 31.936 \\
RAW & -20.236 & -259.496 & -921.179 & 1.148 & 17.813 & 30.989 \\
\hline \hline
\end{tabular}

Source: Authors' results from GT AP simulations

\section{RESUlTS AND ANALYSIS}

After the sensitivity test by simulating the value of antidumping tariff rates for certain duties, it was found that the results showed a high level of stability, so it can be concluded that the simulation result is quite strong. Simulation results for each country (Indonesia and Argentina) can be seen in the following table.

\section{A. 4Changes in Production an Industry Level}

The change in production at the industry level is displayed by Tabel III, where industry sectors are categorized for each region. A mong 12 industry while grain, meat, extraction, and food processing are considered as land and natural resource intensive, textiles, light manufactures, and services are conventionally labor-intensive industries, leaving the remaining industries namely heavy manufactures, utilities, transport and communications to be capital intensive (see Table II for details). The simulation results show that while the land and recourse intensive industries in Indonesia expends, these industries contract in Argentina and AS. A similar pattern occurs in the labor-intensive industries. When these industries expand in Argentina and AS, they contract in Indonesia, indicating a complementary relationship exists between these two countries in line with their distinct comparative advantage.

The US antidumping biodiesel import policy on Indonesian and Argentine impacts on the decline in the biodiesel industry output in both countries. Indonesia's biodiesel industry output decreased from 35.7 to 38.8 percent. While Argentina's biodiesel industrial output decreased from 2.5 to 6 percent. The results of this simulation are in line with the findings of [11] stating the existence of a negative correlation between the import duty of antidumping by the importer and the exporter's industrial output. The decline in the biodiesel industry output in both countries certainly has an impact on the labor market. Given the biodiesel industry in both countries is labor intensive.

The US antidumping biodiesel import policy on Indonesia and Argentina biodiesel have a positive impact on the US biodiesel industry output. The existence of antidumping duty protection increases the output of the US biodiesel industry by 1 to 2 percent. Surely this is in line with the expectations of the US government to protect its domestic producers. This finding is same as [10] study which states that antidumping duty policy increases the output and profit of domestic industries.

\section{B. Change in Regional GDP}

Changes in GDP from 22 regions are shown in Table 4. It can be clearly seen from the table that the imposition of US antidumping duties has a negative impact on changes in GDP of Indonesia and Argentina. This is confirmed by the findings of [16] stating that there is a negative correlation between antidumping duties with the foreign output. In addition, US antidumping duties policy for Indonesia and
Argentina has a negative impact on other biodiesel producing countries such as Australia, Singapore, Korea, Taiwan, China, and Norway. At import tariffs imposed by the US (baseline), GDP Indonesia and Argentina experienced a change of 0.001 percent and 0.01 percent. In the scenario tariffs, 127 percent and 341 percent showed a significant decrease of Indonesia's GDP by 1.4 percent and 1.7 percent, while Argentina 0.2 percent and 0.4 percent. These results suggest that the US is the largest market of biodiesel products for both countries. Also interesting to note, US antidumping duties policy for Indonesia and Argentina has a positive impact on the US economy. The positive impact is indicated by the increase in GDP by 0.013 percent and 0.028 percent (for scenarios tariffs 127 percent, and 341 percent tariff scenarios for Indonesia) and 0.003 percent, and 0.007 percent (for scenarios tariffs 127 percent, and 341 percent tariff scenario for Argentina), In other words, the US benefits from protecting its domestic producers. In addition, some countries exporting biodiesel to the US such as EU, Canada, Panama, Netherlands, Germany, Belgiu m, Finland, Portugal, Spain, North A merica and Latin America countries also benefit from the enforcement of such antidumping.

\section{Change in Economic Welfare}

The impact of changes in Indonesian and Argentine welfare from US antidumping duties policy can be seen from the equivalent variation (EV) (see Table 5 for details). Indonesia and Argentina have significantly decreased welfare. Indonesia decreased by 163.8 US\$ million (baseline), 1813 US \$ million (tariffs 127\%) and 2557.2 US\$ million (341\% tariffs). While Argentina has decreased by 11.8 US $\$$ million (baseline), 210.4 US $\$$ million (tariffs $127 \%$ ) and 4889 US \$ million (341\% tariffs). Significant welfare declines in both countries stem from falling GDP, exports and industrial output. This policy also has a negative impact on Asia-Pacific and EU countries. This shows that US policy has a significant effect on Asia-Pacific countries. Meanwhile, the US has increased welfare significantly. US policy of wearing antidumping duties provides benefits for the US especially domestic biodiesel industry. This can be seen from the increase in GDP and domestic biodiesel industry output.

\section{CONCLUSION}

This study aims to analyze the impact of US antidumping import tariffs on the economies of Indonesia and Argentina. Although still tentative, our results show that Indonesia and Argentina suffered significant losses. Evidently, the imposition of antidumping duties for biodiesel products of both countries caused a significant reduction of industrial output, exports, GDP and welfare. This confirms that America is a large market for the second biodiesel product. In addition, our results indicate that the policy has a positive impact on the US economy, especially the domestic 
biodiesel industry. Reflected from the rise in industrial output, exports, GDP and US welfare. It is also important to note that imposition of US anti-dumping duties leads to welfare declines in Asia-pacific and EU countries. In this paperless explains the deeper causes of the decline of welfare in Asia-Pacific and EU countries. This needs to be the next research.

\section{REFERENCES}

[1] H. Zhang, "Political connections and antidumping investigations: Evidence from China," China Economic Review, pp. 1-7, 2018.

[2] T. J. Prusa and S. Skeath, "The economic and strategic motives for anti-dumping filings," NBER Working Paper, No.8424, 2001.

[3] D. Salvatore, "A model of dumping and protectionism in the United States," Weltwirtschaftliches Archiv, pp.763-781, 1989.

[4] Komite Antidumping Indonesia, "Sambutan Ketua Kadi," Kementerian Perdagangan Republik Indonesia, 2018.

[5] S. K. Singh, "An analysis of anti-dumping cases in india," Economic and Political Weekly, pp. 1069-1074, 2005.

[6] P. K. M. Tharakan and J. Waelbroeck, "Anti-dumping and countervailing cuty decisions in the EC and in the US," European Economic Review, pp. 171-93, 1994.

[7] S. Budi, "Amerika dan Kebijakan Ekonomi Trum, p yang Mengkhawatirkan". Geotimes, 2017.

[8] M. J. Kurniasih, "Ketentuan AS Soal Antidumping, Kemendag: Ekspor Menghadapi Tantangan Berat," Okezone, 2016

[9] Gapki, "Kebijakan Subsidi Biodiesel Amerika Serikat dan Tuduhan Dumping Biodiesel Sawit Indonesia" Gapki, 2018.
[10] M. Webb, "The ambiguous consequences of anti-dumping laws". Economics Inquiry, pp. 437-448, 1992.

[11] J. Fender and K. C. Yip, "Tariffs and exchange rate dynamics redux," Journal of International Money and Finance, PP. 633-655, 2000.

[12] J. Konings and H. Vandenbussche, "Antidumping protection hurts exporters: firm-level evidence," paper presented at European Research Workshop in International Trade, pp. 16-18, June 2010.

[13] C. P. Bown and M. A. Crowley, "Trade deflection and trade depression," Journal of International Economics, pp. 176-201, 2007.

[14] D. Vanzetti, K. Christian, and P. Ralf, "Non-tariff measures and regional integration in ASEAN," Contributed paper at the Twenty First Annual Conference on Global Economic Analysis, Cartagena, Colombia, June 13-15,2018.

[15] C. Qi and J. X. Zhang, "The economic impacts of the China-Australia free trade agreement - A general equilibrium analysis," China Economic Review, pp. 1-11, 2018.

[16] C. F. Lee and T. L. Xi, "The effects of antidumping duties in a new open economy macroeconomics model," International Joumal of Economics and Financial,pp. 837-844, 2016.

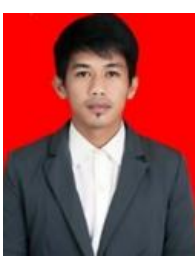

Salman Samir was born on November 3, 1992 in Baranti, Indonesia. Im graduated at the Faculty of Economic, University of Hasanuddin, Indonesia in 2015 with the specialization in economics. From 2016-now is a students of the Master of Science Program at the Faculty of Economics and Business, Gadjah Mada University, Indonesia. Research interests; international trade and public economics 